\title{
The Impact of Parity on the Number of Ovarian Cortical Inclusion Cysts
}

\author{
Leila Sadeghi ${ }^{1}$, Ali Dastranj ${ }^{2}$, Parvin Mostafavi Gharabaghi ${ }^{*}$, Farzam Sheikhzadeh ${ }^{3}$, Sharareh Zamanvand ${ }^{3}$, \\ Mehri Jafari-Shobeiri ${ }^{1}$
}

\begin{abstract}
Objectives: Ovarian cancer is the most lethal gynecologic cancer. Understanding the origin and pathogenesis of epithelial ovarian cancers is one of the most challenging issues of gynecology. According to one of the theories, ovarian cortical inclusion cysts can cause epithelial ovarian cancer, but there are a lot of criticisms against the theory. In this study, the effect of oral contraceptive pills (OCP) on the number and type of inclusion cysts were investigated. Parity is a strong factor in the prevention of ovarian epithelial cancer. The base of this study is that if parity reduced the total number of cortical inclusion cysts (CIC) and tubal metaplasia, the theory would be strengthened.

Materials and Methods: One hundred twenty-five patients under the total abdominal hysterectomy and bilateral salpingooophorectomy (TAH \& BSO) due to causes other than conflict ovaries were considered for study. The patients were categorized in three groups based on their parity. Slides related to normal ovaries were considered for study by pathologist and the results analyzed. Results: The one-way analysis of variance (ANOVA) of total number of cysts revealed a statistically significant main effect, Welch's $\mathrm{F}(2,55.09)=3.51, P<0.05$, indicating that not all groups had the same number of total number of cysts. Also, the results indicated that patients with $0-2$ parity had a significantly higher total number of cysts than patients with 6 and over $(P<0.05)$. Moreover, the results of Games-Howell post hoc procedure indicated that patients with 0-2 parity had a significantly higher number of inclusion cysts than patients with 6 and over $(P<0.05)$.

Conclusion: Based on the results of this study, parity has significant effect on reducing total number of inclusions cysts, but number of tubal type inclusion cyst was not different.

Keywords: Cysts, Epithelium, Ovary, Parity
\end{abstract}

\section{Introduction}

Ovarian cancer is the fifth most common cause of death from cancer in women. In ovarian cancer, the highest proportion of fatality per one case is seen among all genital cancers. A woman with probability of $1 \%-1.5 \%$ faces the risk of ovarian cancer at some point in her lives. The risk of dying from ovarian cancer per woman is about $0.5 \%$ (1).

The peak incidence of invasive epithelial ovarian cancer is age 56-60 years. This cancer is relatively uncommon in women under 45 years (2).

Unfortunately, so far no progress has occurred in screening and early diagnosis of the ovarian cancer and understanding its pathogenesis. Investigator believes that determination of the source of the malignancy can help in screening and early diagnosis. (2)

For many years the hypothesis that epithelial ovarian cancer originates from ovarian cortical inclusion cysts (CIC) is lived. According to this hypothesis, cells-from ovarian surface epithelium (OSE) - that line these cysts multiply and mutate in response to bio-active factors and oncogenes (3).

Another interesting theory in recent decades is that serous ovarian cancer originates from tubal intra epithelial neoplasia (serous tubal intraepithelial cancer, STIC). Despite the overwhelming evidence in favor of the second theory, investigators failed to put the OSE theory aside (4). In this connection, there is much evidence; for example, metaplastic changes in epithelial inclusion cysts, from one type of epithelium to another, which can predispose to neoplastic changes (5).

Ovarian cancer is associated with infertility and low parity. The most important factors are the duration of fertility and reproductive history (6). Every pregnancy reduces the risk of ovarian cancer by about $10 \%$.

Protection against ovarian cancer is one of the main advantages of oral contraceptive pills (OCP). The risk for all histologic type of ovarian cancer is reduced by $40 \%$ in OCP users compared with women who do not use this method. Many of these risk-reducing factors such as OCP and parity verify frequent ovulation hypothesis as etiology of ovarian cancer. According to this hypothesis, the main cause of ovarian cancer is dysfunction in the process of repairing the surface epithelium (7).

Accordingly, the effect of parity on the number of inclusion cysts and tubal metaplasia of the cyst are investigated.

Received 19 May 2015, Accepted 4 September 2015, Available online 21 September 2015

${ }^{1}$ Department of Obstetrics and Gynecology, Women's Reproductive Health Research Center, Tabriz University of Medical Sciences, Tabriz, Iran. ${ }^{2}$ Women's Reproductive Health Research Center, Tabriz University of Medical Sciences, Tabriz, Iran. ${ }^{3}$ Animal Sciences Group, Faculty of Natural Sciences, University of Tabriz, Tabriz, Iran.

*Corresponding author: Parvin Mostafavi Gharabaghi, Department of Obstetrics and Gynecology, Women's Reproductive Health Research Center, Tabriz University of Medical Sciences, Tabriz, Iran. Tell: 0098-41-35541221, Email: pm_gharabaghi@yahoo.com 
It is believed that if cortical inclusion cyst is the source of epithelial ovarian tumors, high number of pregnancies would reduce the number of cysts and reduce tubal metaplasia.

Three theories have been proposed in regard to the origin of ovarian cancer:

1) The theory of OSE: According to this theory, OSE is origin of most ovarian stromal and epithelial cancers and can be differentiated to tissues with Mullerian types and create ovarian epithelial cancer (8). When culturing purified human OSE, it is converted to aggressive neoplasms similar to high-grade and low- undifferentiated grade serous carcinomas (9).

2) Theory of STIC: In this theory, the origin of high-grade ovarian serous tumors is malignant lesion in the fimbrial epithelium (10).

3) The theory of junction location of tubal fimbria epithelium with peritoneum (tubo-peritoneal junction [TPJ]): This location is the hot spot of the female genital tract for the generation of tumors (11).

The purpose of this study is to evaluate the effect of the number of pregnancies as one of the reducing decisive factors for ovarian cancer on the number of inclusion cysts in superficial epithelium as the source of ovarian tumors.

\section{Materials and Methods}

The study is descriptive analytic. Women who underwent hysterectomy and oophorectomy in Zahra hospital during 2011-2013 were included. For this purpose, the hospital records of patients who underwent hysterectomy and oophorectomy from any cause had been studied and those with normal ovaries were selected. The questionnaire was filled for each patient according to the data. In the case of incomplete records they were called. Patients with accurate information about the age, number of pregnancies, number of delivery and type of contraceptive method and were divided into three main groups based on the type of contraceptive methods:

1. Patients who had 2 pregnancies over 20 weeks.

2. Patients who had between 3 and 5 pregnancies over 20 weeks.

3. Patients who had at least six pregnancies over 20 weeks.

Patients who had normal ovaries in the final pathology report and their ovarian size were normal were selected. The paraffin blocks of patients' ovarian tissue were obtained from the pathology archive of the hospital; from the paraffin blocks, sections with a thickness of 5 micrometers were prepared. Then, after consolidating the tissue in neutral buffered formalin and staining with hematoxylin and eosin, routine histological examination was carried out.

Slides obtained by a woman pathologist were reviewed to determine the presence of ovarian surface epithelial in obtained slices, the number of ovarian cortical cysts and existing tubal cell types in cyst epithelium and OSE. The pathologist, during examination of the slides, was not aware of the group of each patient.

For analysis of morphological results in the experimental groups, SPSS software, binomial regression analysis and chi-square test were used. Corresponding graphs were plotted by Excel software. The results were presented as standard deviation and the values of $\mathrm{P}<0.05$ were considered statistically significant.

\section{Results}

In order to compare bilateral oophorectomy with unilateral oophorectomy, the mean number of cysts (both normal and tubal) was analyzed. This means for those with a decimal number less than 5 was rounded to the larger number and for people with an average of more than 5 was rounded to smaller number.

\section{Demographic Characteristics}

The mean age of the 187 studied patients was $51.67 \pm 7.5$ years. The mean age in patients with number of parity 0-2 was $49.77 \pm 6.5$, with number of parity $3-5$ was $49.36 \pm 6.5$ and with number of parity 6 and over was $68.64 \pm 6.5$, respectively. Statistical analysis ( $t$ test) of mean age between the two groups did not show significant difference $(P=0.9)$.

Figures $1 \mathrm{~A}$ and $1 \mathrm{~B}$ show microscopic images of the OSE with normal and tubal cells. Figures $1 \mathrm{C}$ and $1 \mathrm{D}$ show microscopic images of the cortical ovarian cysts with normal and tubal cells.

A one-way analysis of Variance (ANOVA) was used to examine the question of whether three groups of patients with number of parity $0-2,3-5$, and 6 and over differ with respect to their total number of cysts. Since the Levine's $F$ test revealed that the homogeneity of variance assumption was not met $(P=0.001)$, the Welch's $\mathrm{F}$ test was used. An alpha level of .05 was used for all subsequent analyses.
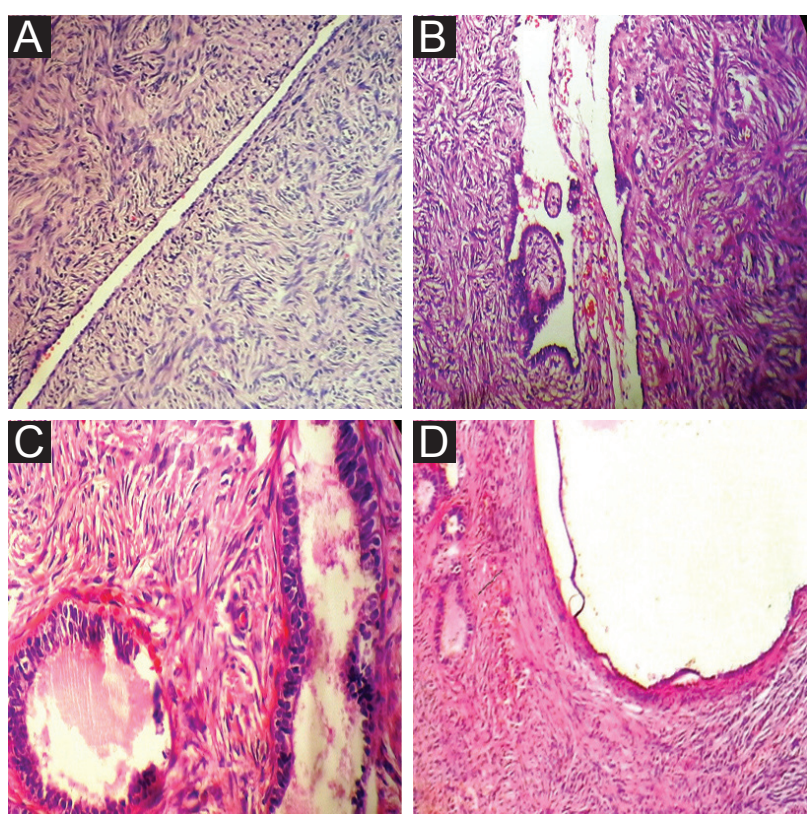

Figure 1. A) Microscopic Image of the Ovarian Surface Epithelium (OSE) With Normal Cell. B) Microscopic Image of OSE With Tubal Cells. C) Microscopic Image of Cortical Ovarian Cysts With Tubular Cells. D) Microscopic Image of Cortical Cysts With Normal and Tubular Epithelium (H \& E, X320). 
The one-way ANOVA of total number of cysts revealed a statistically significant main effect, Welch's $F(2,55.09)=$ $3.51, P<0.05$, indicating that not all groups had the same number of total number of cysts. Post hoc comparisons, using the Games-Howell post hoc procedure, were conducted to determine which pairs of the three groups differed significantly. The results indicated that patients with $0-2$ parity had a significantly higher total number of cysts than patients with 6 and over $(P<0.05)$. Figure 2 illustrates that patients with six and more parities had lower number of cysts compared to groups with lower parity. Another one-way ANOVA was employed to examine the difference between three groups of patients categorized according to their number of parity, in number of inclusion cysts. Since the assumption of homogeneity of variance was violated $(P=0.001)$, the Welch's F test was used. The results of oneway ANOVA revealed a statistically significant differences between three groups in terms of number of inclusion cysts, Welch's $\mathrm{F}(2,55.89)=3.48, P<0.05$. As depicted in Figure 3, the results of Games-Howell post hoc procedure indicated that patients with 0-2 parity had a significantly higher number of inclusion cysts than patients with 6 and over $(P<0.05)$.

The Effect of Parity on the Number of Tubal Cysts Due to the nature of the data that it had over distribution, to examine the impact of group on the number of tubal

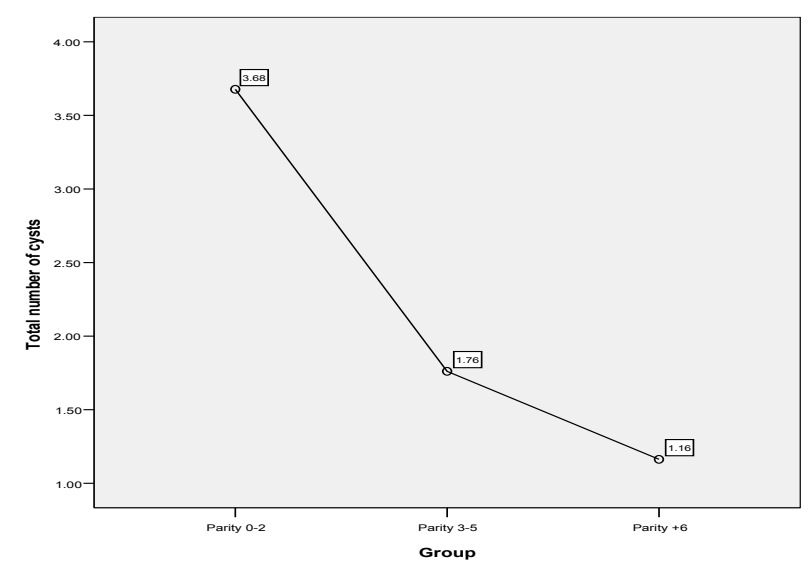

Figure 2. Total Number of Cysts According to Number of Parity.

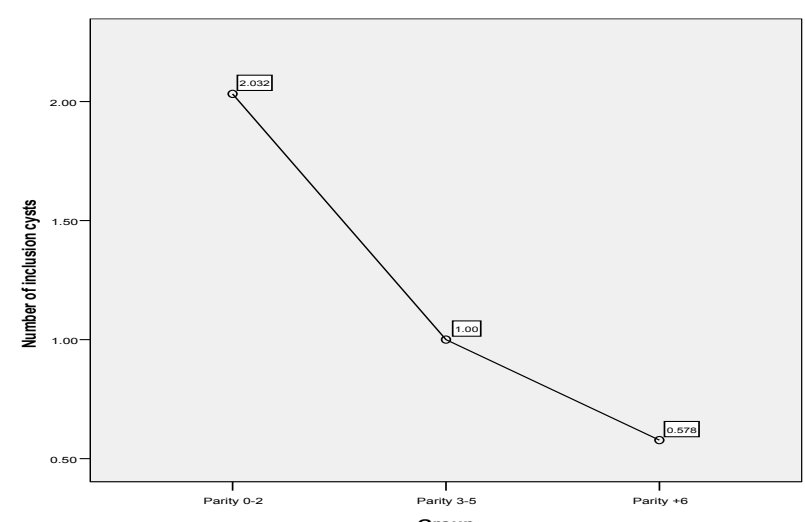

Figure 3. Number of Inclusion Cysts According to Number of Parity. cysts, the negative binomial regression model was utilized. According to the results parity did not have significant impact on the number of tubal cysts.

\section{Discussion}

Ovarian cancer is the fifth most common cause of death due to cancer in women. Ovarian cancer is called "silent killer," as it has no symptoms and is detected at advanced stages (1).

The discussion about the pathogenesis and origin of epithelial ovarian cancer is still one of the hottest and most challenging topics in pathological obstetrics. Fortunately, in recent years, there have been major advances in this matter which can improve our understanding of the pathogenesis of ovarian cancer and it can certainly lead to advances in prevention, screening and early detection of this disease. The question is that whether ovarian cancer and in particular High Grade Serous Carcinoma (HGSC) originate from OSE, CIC and/or fallopian tubal epithelium (especially fimbria).

Until recently, the prevailing view was that these tumors originate from OSE, malignant transformation in OSE or CIC. In fact, despite the fact that these tumors are mainly epithelial, recently, histological studies on HGSC present compelling evidence that these tumors originate from the fimbrial mucosa of fallopian tubes.

Briefly based on STIC theory, the high grade ovarian serous cancer HGSC are not primary ovarian neoplasms, but metastases from fallopian tube to the ovary.

Although there is much evidence in favor of this theory, but STIC is associated with only $60 \%$ of these neoplasms and the question arises that where is the origin of remaining $40 \%$ (12)?

We actually planned to answer this question. In fact, we believe that even though there are arguments in favor of origin of HGSC from STIC, but the OSE and ovarian CIC as a source of additional and perhaps important neoplasms cannot be ignored. If CIC are the origin of ovarian epithelial cancer, proved risk factors for ovarian cancer should increase the number of cysts and increase the tubal metaplasia in cysts and in OSE. This attitude can be subject for extensive research to find the origin of this malignancy. It is not so far-fetched that age increases the numbers of ovarian inclusion cysts, as well as an increase in tubal type cysts.

Banet and Kurman in a study in 2014 analyzed the relationship between aging and the number of CIC in the ovaries. They used PAX8 as an indicator of Mullerian (tubal type cells) and calretinin as mesothelial indicator (Flat cells). They did not find CIC with tubal type epithelium in pre-menarche girls. They found a significant correlation between tubal type CIC that increases with aging. These findings mean that the existence of two types of CIC (one flat epithelium and the other one with columnar and tubal view epithelium) and the absence of CIC with tubal epithelial in the ovaries of the girls before menarche give ground for following finding: ciliary pattern of CIC is formed over time with the process of ovulation and im- 
plantation of fimbrial cells in ruptured area of OSE during ovulation (13). If the findings of Banet and Kurman were correct, then, parity must decrease the number of inclusion cysts and especially, the cysts with tubal epithelial lining. In our study the total number of inclusion cysts in group with the parity number more than 6 inclusion cysts was significantly lower. But number of tubal type inclusion cysts was same.

Auersperg et al showed that OSE normal cells, express markers of stem cells. The OSE in response to changes in the microenvironment and different growth factors can convert into different types of cells (14). The process of metaplasia from flat or cubic cells of CIC and OSE to cylindrical cells similar to fimbria epithelium is approved by IHC not only morphologically but also functionally (15). The culturing of OSE cells in the culture medium with endometrial stroma and estrogen cause endometrioid metaplasia in OSE (16).

\section{Conclusion}

Based on the results of this study, ovarian CIC were significantly lower in the group with more than 6 parity. Types of tubal inclusion cysts were not significantly different between the 3 groups. Ovarian surface epithelium in all groups was same. Normal and tubal type OSE was not significantly different. Our study was based on morphological features only.

For a better survey we should assess tissue specific markers such as calretinin and PAX8. In the current study we included patients who use OCP for 3 years or less than 3 years. Due to the low number of samples, the number of cysts in both groups showed no significant differences, probably because we had samples (patients) that had used OCP for less than 5 years and this distorted the study finding. Another study should be arranged over samples (patients) who have not used OCP in order to look closely at the impact of parity on the OSE.

\section{Ethical Issues}

Since the research and laboratory works were performed over pathology samples after surgery, there was no need to get informed consent. No additional cost was imposed to the patients. All information about patient records and phone calls was kept confidential.

\section{Conflict of Interests}

The authors declare no conflict of interests.

\section{Financial Support}

The researchers received no financial support or grant from any funding agency in the public and commercial sectors.

\section{References}

1. Berek J, Longacre, Fried Lander M. Ovarian Fallopian Tube and Peritoneal Cancer. In: Berek \& Novak Gynecology. 15th ed. Philadelphia: Williams \&
Wilkins; 2013.

2. Kurman RJ. Origin and molecular pathogenesis of ovarian high-grade serous carcinoma. Ann Oncol. 2013;24 Suppl 10:x16-21. doi:10.1093/annonc/mdt463.

3. Yildiz I, Tas F, Kilic L, $t$ al. A high serum level of M65 is associated with tumour aggressiveness and an unfavourable prognosis for epithelial ovarian cancer. Cancer Chemother Pharmacol. 2013;72(2):437-444. doi:10.1007/s00280-013-2212-z.

4. Nik NN, Van R, Shih IeM, Kurman RJ. Origin and pathogenesis of pelvic (ovarian, tubal, and primary peritoneal) serous carcinoma. Annu Rev Pathol. 2014;9:27-945. doi:10.1146/annurevpathol-020712-163949.

5. Li HX, Lu ZH, Shen K, et al. Advances in serous tubal intraepithelial carcinoma: correlation with high grade serous carcinoma and ovarian carcinogenesis. Int J Clin Exp Pathol. 2013;7(3):848-857.

6. Lim D, Oliva E. Precusors and pathogenesis of ovarian carcinoma. Pathology. 2014;45(3):229-242. doi:10.1097/ PAT.0b013e32835f2264.

7. Hatzipetros I, Gocze PM, Farkas B. Oral contraceptive pills as primary prevention for ovarian cancer: a systematic review and meta-analysis. Obstet Gynecol. 2013;122(5):1114.

8. Kurman RJ, Shihie M. The origin and pathogenesis of epithelial ovarian cancer: a proposed unifying theory. Am J Surg Pathol. 2012;34(3):433-443. doi:10.1097/ PAS.0b013e3181cf3d79.

9. Bowen NJ, Walker LD, Matyunina LV, et al. Gene expression profiling supports the hypothesis that human ovarian surface epithelia are multipotent and capable of serving as ovarian cancer initiating cells. BMC Med Genomics. 2009;2:71.

10. Erickson BK, Conner MG, Landen CN Jr. The role of fallopian tube in the origin of cancer. Am J Obstet Gynecol. 2013;209(5):409-414.

11. Seidman JD, Yemelyanova A, Zanio RJ, Kurman RJ. The fallopian tube-peritoneal junction: a potential site of carcinogenesis. Int J Gynecol Pathol. 2011;30(1):4-11.

12. Auersperg N. Ovarian surface epithelium as a source of ovarian cancer: Unwarranted speculation or evidencebased hypothesis? Gynecol Oncol. 2013;130(1):246-251.

13. Banet N, Kurman RJ. Two types of ovarian cortical inclusion cysts: Proposed origin and possible role in ovarian serous carcinogenesis. Int J Gynecol Pathol. 2014;34(1):3-8.

14. Auersperg N, Wong AS, Choi KC, Kang SK, Leung PC. Ovarian surface epithelium: biology, endocrinology, and pathology. Endocr Rev. 2011;22(2):55-88.

15. Auersperg N. The origin of ovarian carcinomas: a unifying hypothesis. Int J Gynecol Pathol. 2011;30(1):1221.

16. Okamura H, Katabuchi H, Nitta M, Ohtake H. Structural changes and cell properties of human ovarian surface epithelium in ovarian pathophysiology. Microsc Res Tech. 2006;69(6):69-81. provided the original work is properly cited. 